\title{
Isolation, Purification and Anticancer Effect of Hydatid
}

\author{
Lei Zhang ${ }^{1,2}$, Lizhong Guo ${ }^{1}$, He Qian ${ }^{3,4,5}$ \\ ${ }^{1}$ Shanghai Sun Avenue Biotechnology Co.,LTD. \\ ${ }^{2}$ State key laboratory of food science and technology, Jiangnan University. \\ ${ }^{3}$ School of food science and technology, Jiangnan University. \\ ${ }^{4}$ International joint laboratory for food safety, Jiangnan University. \\ ${ }^{5}$ School of life science, Qingdao agricultural university
}

Keywords: Hydatid; polysaccharide; separation and purification; anticancer effect

\begin{abstract}
Hydnisella polysaccharides were extracted from the fruiting bodies of Hydrangea, and the crude polysaccharides of Hydatid were extracted by water extraction and alcohol precipitation. The purified polysaccharides were purified by DEAE-52 cellulose chromatography column. The Hep-G2 cells were cultured and the anticancer activity of the samples was studied by MTT assay. The results showed that the best inhibitory effect on Hep-G2 cells was $0.01 \mathrm{mg} / \mathrm{mL}$ boiled supernatant, the inhibition rate was $19.21 \%$, and the positive control group was $29.56 \%$. Therefore, Hydatid polysaccharide has a good inhibitory effect on hepatoma cell Hep-G2and plays an important role in drug selection in anticancer effects.
\end{abstract}

Hydrangea [Sparassis crispa (Wulf.) Fr.] is also known as the flower mushroom, belonging to the non-Pleurotus, Hydrangea, Hydrangea ${ }^{[1]}$. At present, a number of studies have confirmed that Hydatid has good anti-oxidation ${ }^{[3]}$, improved immunity ${ }^{[4]}$, anti-tumor activity ${ }^{[5]}$ and other effects, and the anti-tumor activity of Hydatid is mainly the polysaccharide in its fruit body. In function, mainly by stimulating the production of cytokines to fight tumors ${ }^{[6]}$. Due to the excellent efficacy of Hydrangea, it has gradually become a research hotspot in recent years. South Korea and Japan have the most in-depth research on it, while the research on the efficacy of Hydatid in China focuses on antioxidants and immunity. Anticancer effects have rarely been reported.

On the one hand, this study used the alcohol precipitation water extraction method to purify and separate it with DEAE-52 cellulose chromatography, which greatly improved the polysaccharide yield and purity. On the other hand, the anti-tumor and immunomodulatory activities of Hydatid polysaccharides were evaluated in vitro, and it is of great significance to study the anti-tumor effect and mechanism of Hydatid polysaccharides in one stepand to explore new drugs and new treatment methods for preventing and treating tumors.

\section{Materials and methods}

\subsection{Experimental materials}

Hydrangea fruit body dry powder (provided by Shanghai Hexian Mushroom Biotechnology Co., Ltd.). Human hepatoma cell line HepG2. RPMI-1640 medium, cell digestive juice: $0.25 \%$ trypsin, D-Hanks solution, streptomycin solution, MTT, fluorouracil. Sevage reagent (n-butanol: chloroform $=1: 4$ ) reagents such as acetone are of analytical grade (Zhongguo Group Chemical Reagent Co., Ltd.). Centrifuge (Shanghai Anting Scientific Instrument Factory). UV-1800 UV spectrophotometer (Shimadzu Corporation, Japan). DEAE-52 cellulose chromatography column $(2.6$ x $40.0 \mathrm{~cm})$. Electrophoresis unit (Clever Ltd., UK).

\subsection{Experimental methods}

\subsubsection{Separation of polysaccharides from the genus Hydrangea}

In this paper, the alcohol-sinking water combined with the Sevage method ${ }^{[11]}$ was used to 
separate and extract the polysaccharides of the genus Hydrangea. Hydrangea fruit body dry powder plus $95 \%$ ethanol, $100^{\circ} \mathrm{C}$ water bath, $1.5 \mathrm{~h}$. Discard the supernatant and dilute with distilled water for $3 \mathrm{~h}$. The resulting solution was coarsely filtered with gauze and centrifuged (4500 rpm, $6 \mathrm{~min}$ ) to obtain a supernatant for use. The residue was boiled with distilled water for $3 \mathrm{~h}$, centrifuged, and the precipitate was discarded. The obtained supernatants were combined twice, concentrated in a water bath at $70^{\circ} \mathrm{C}$, concentrated, cooled to room temperature, added with ethanol, and condensed in a refrigerator at $4^{\circ} \mathrm{C}$ for $12 \mathrm{~h}$. The resulting solution was centrifuged (10000 rpm, $10 \mathrm{~min}$ ), washed with absolute ethanol, acetone and diethyl ether to give a crude polysaccharide.

\subsubsection{Purification of polysaccharides from the genus Hydrangea}

The method of separating and purifying polysaccharides from Inonotus obliquus by Dong Wenxia $^{[12]}$ et al. DEAE-52 cellulose static method was used to dissolve the crude polysaccharide powder in appropriate distilled water and added to DEAE-52 cellulose chromatography column (2.6×40.0). Chromatography was carried out in $\mathrm{cm}$ ), and elution was carried out using 0 to $2 \mathrm{M}$ sodium chloride. The eluate of each gradient was collected and the polysaccharide concentration was followed by phenol sulfuric acid method to determine the elution endpoint.

\subsubsection{Purity testing of polysaccharides from the genus Hydrangea}

In this paper, the purity of polysaccharides from the genus Hydrangea was detected by the phenol-sulfuric acid method ${ }^{[13]}$. Take $1 \mathrm{ml}$ of each sample solution in a test tube, replenish water to 2 mland allow to stand at room temperature (about 30 minutes). The solution of the standard glucose solution in a $0.00 \mathrm{ml}$ tube was used as a reference solutionand the optical density OD value was measured at a maximum absorption wavelength of $490 \mathrm{~nm}$.

\subsubsection{SDS-polyacrylamide gel electrophoresis (SDS-PAGE) analysis}

SDS-PAGE gel electrophoresis ${ }^{[14]}$ : $16 \mu \mathrm{L}$ sample was added to $5 \times$ SDS-PAGE Loading Buffer 4 $\mu \mathrm{L}$, heated at $100^{\circ} \mathrm{C}$ for $3 \mathrm{~min}$, centrifuged at $8000 \mathrm{rpm}$ for $1 \mathrm{~min}$ at $4^{\circ} \mathrm{C}$, the supernatant was collected, placed on iceand the whole sample was processed. Thus, $20 \mu \mathrm{L}$ of the supernatant of the prepared sample was added to the spotting well.

\subsubsection{Cell culture and counting}

The human hepatoma cell line HepG2, SMMC-7721 was separately cultured in a cell culture medium containing $10 \%$ fetal calf serum. All the cells in the exponential growth phase were cultured in an incubator at $5 \% \mathrm{CO} 2,37^{\circ} \mathrm{C}$ and saturated humidity. When the cell fusion state reached $80 \%$ to $90 \%$, they were digested with $0.25 \%$ trypsin and passaged. Count with a cell counting plate according to the white blood cell counting method.

\subsubsection{MTT (Methyl thiazolyl tetrazolium) colorimetric analysis}

On the ultra clean worktable, trypsin is digested and repeatedly blown into a single-cell suspension, whose cell concentration is adjusted into $1 \times 10^{6} / \mathrm{mL}$ with fresh RPMI-1640 culture solution. Tumor cells are placed in a 96-well culture plate with a dose of $150 \mu \mathrm{L}$ per well, which is put into incubator with $5 \% \mathrm{CO}_{2}$ at $37^{\circ} \mathrm{C}$ under saturated humidity. $50 \mu \mathrm{L}$ sample to be tested is added into each well for processing after 24 hours. $20 \mu \mathrm{LMTT}$ is added into each well after 48 hours. After 4 hours of culture, the supernatant medium solution was carefully removed, with $150 \mu \mathrm{L}$ dimethyl sulfoxide (DMSO) added to each hole. Then it is put into the oscillator for 10 minutes, and the absorbance is measured at a wavelength of $570 \mathrm{~nm}$ using an enzyme marker.

Cell growth inhibitory rate $(\%)=(1-$ OD value of drug group/OD value of negative control group) $\times 100 \%$

\subsection{Data processing}

After three parallel trials in five groups, Origin 7.5 was used to plot the data through SPSS statistical software (SPSS 21.0, American SPSS company) and data statistics and analysis was conducted at the significance level of $\mathrm{p}=0.05$. 


\section{Result and analysis}

\subsection{Separation of polysaccharides from Hydrangea spore}

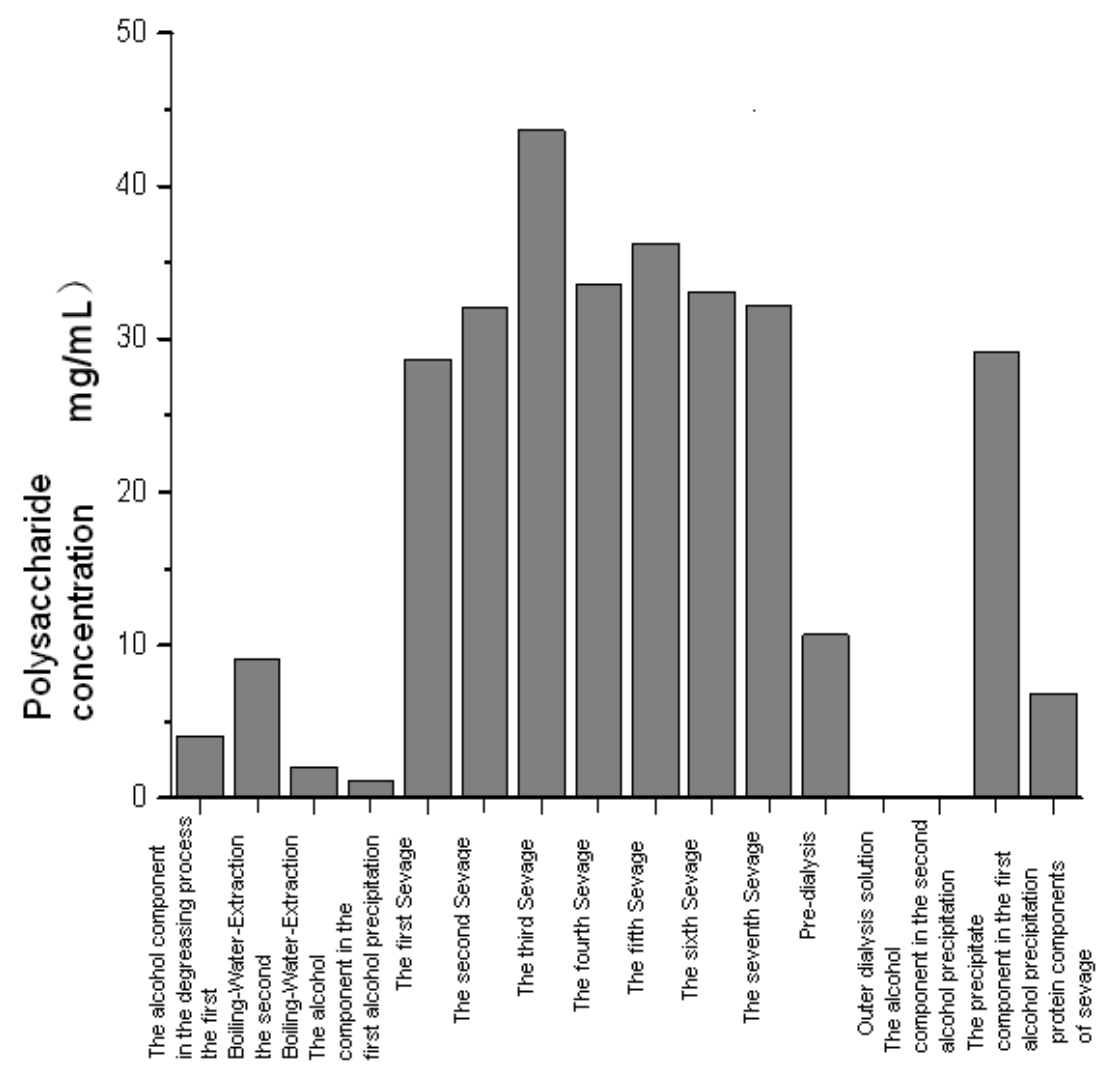

Figure 1. Variation of polysaccharide concentration in the process of polysaccharide separation

As shown in figure 1, the polysaccharide concentration is not completely linear. The polysaccharide concentration of the precipitated components in the seven Sevage and the first alcohol precipitation was higher while there was no polysaccharide in the alcohol components of the dialysate and the second alcohol precipitation.

\subsection{Purification of polysaccharides in Hydrangea spore}

Table 1. Results of DEAE-52 cellulose chromatography

\begin{tabular}{lccccc}
\hline & $\begin{array}{c}\text { NaClconcentration } \\
(\mathrm{mol} / \mathrm{L})\end{array}$ & $\begin{array}{c}\text { Total weight of } \\
\text { the solid(g) }\end{array}$ & $\begin{array}{c}\text { Polysaccharide } \\
\text { concentration }(\mathrm{mg} / \mathrm{mL})\end{array}$ & $\begin{array}{c}\text { Total weight of the } \\
\text { polysaccharide(g) }\end{array}$ & Purity \\
\hline 0.0 & 0.1166 & 0.006745 & 0.1012 & $86.77 \%$ \\
& 0.2 & 0.3305 & 0.009232 & 0.2769 & $83.80 \%$ \\
& 0.5 & 0.0739 & 0.003462 & 0.0519 & $70.27 \%$ \\
& 0.8 & 0.0445 & 0.001851 & 0.0278 & $62.40 \%$ \\
& 1.0 & 0.0296 & 0.001312 & 0.0197 & $66.49 \%$ \\
& 2.0 & 0.0133 & 0.000672 & 0.0101 & $75.77 \%$ \\
Total weight & & & & & \\
Gross recovery & & 0.6084 & & & \\
Recovery of & & $59.92 \%$ & & & \\
polysaccharides & & $82.86 \%$ & & & \\
\hline
\end{tabular}

The results of deae-52 cellulose chromatography are shown in Table 1 . When the $\mathrm{NaCl}$ gradient was $0.0 \mathrm{~mol} / \mathrm{L}$, the purity was the highest. After the purification of the polysaccharides by $6 \mathrm{NaCl}$ gradients, the purity reached $80.14 \%$, which was a significant increase compared with before. 


\subsection{Analysis of SDS- polyacrylamide gel electrophoresis (SDS-PAGE)}

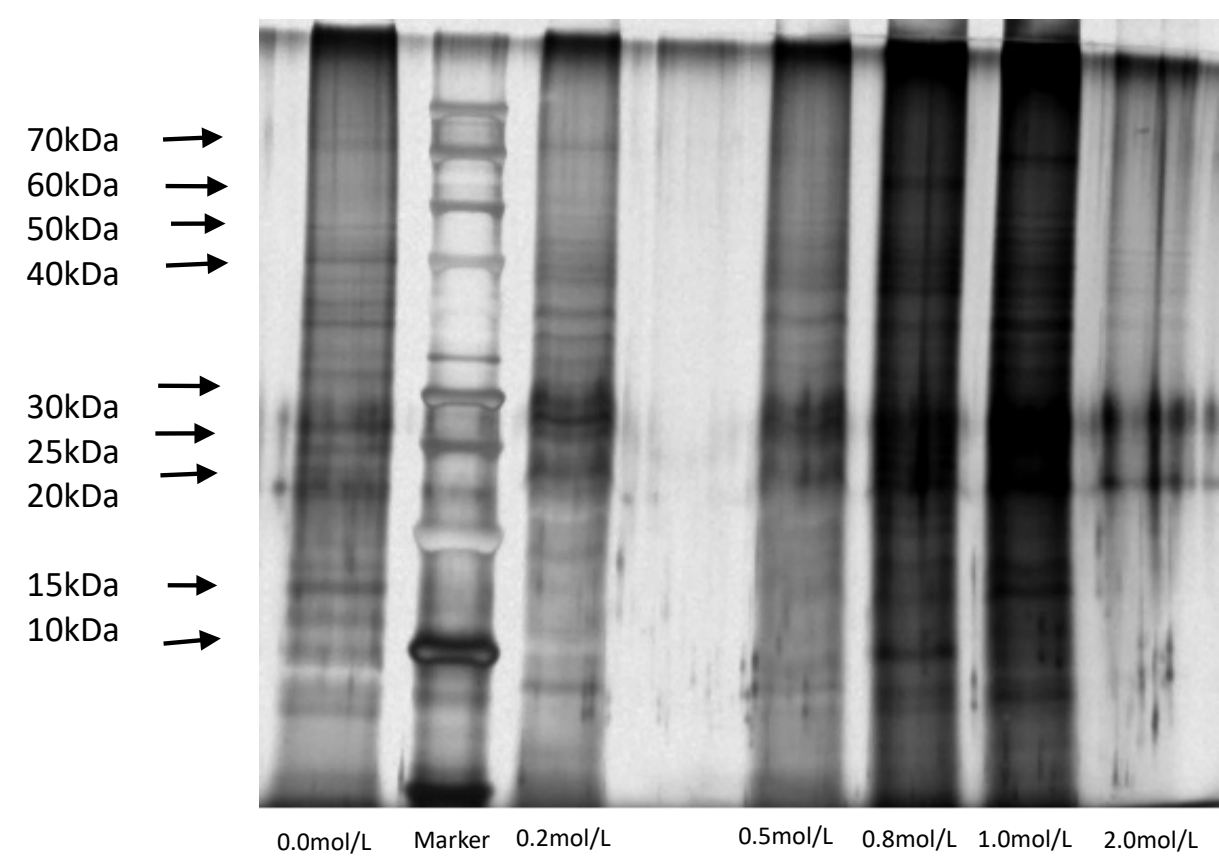

Figure 2 SDS-PAGE result

SDS-PAGE analysis was performed on six samples of polysaccharides obtained by DEAE-52 cellulose chromatography. As can be seen from Figure 2, there are 2 strips on results of the six groups of samples, with similar size at about 25KDa 30KDa.

\subsection{Inhibition on hepatocellular carcinoma cells Hep-G2 by polysaccharides}

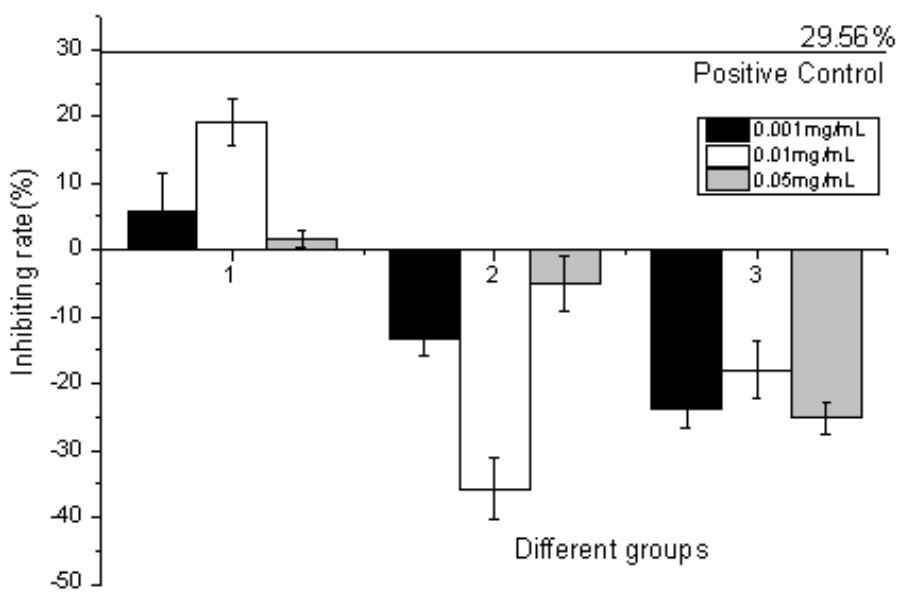

Figure 3. Inhibition on hepatocellular carcinoma cells Hep-G2 by polysaccharides

(1. Supernatant in Boiling-Water-Extraction 2. Supernatant in Sevagedeproteinization 3.

DEAE-52Cellulose purified eluate ( NaClgradient: $0.2 \mathrm{~mol} / \mathrm{L}$ )

As shown in figure 3, the inhibition rate on Hep-G2 hepatocellular carcinoma cells was 5.74\%, $19.21 \%$ and $1.68 \%$ in the first sample with low, medium and high concentration. According to the analysis of variance, the inhibitory effect of the samples with low or high concentration on the hepatoma cells was significantly different from that of the positive control at the $1 \%$ level, and this group of samples showed a certain inhibitory effect on hepatocellular carcinoma cells, which was the strongest at the medium concentration of $0.01 \mathrm{mg} / \mathrm{mL}$. Compared with the positive control, the anti-cancer effect of this sample was greater and could be used as anticancer drug. The inhibition 
rate on Hep-G2 hepatocellular carcinoma cells was negative at three different concentrations in both sample 2 and sample 3, showing no obvious inhibition effect on hepatocellular carcinoma cells and not suitable as an anticancer drug.

\section{Conclusion}

Sparassis crispa was extracted by water extraction and alcohol precipitation. Further studies on anticancer activity show that the best inhibition effect on Hep-G2 hepatocellular carcinoma cells was $0.01 \mathrm{mg} / \mathrm{mL}$ of Hydrangea fungus spore dry powder boiled on the supernatant liquid, with an inhibition rate of $19.21 \%$. Therefore, active ingredient in the anticancer activity of Sparassis crispa has been further confirmed and plays an important role in the clinical application of anticancer drugs.

\section{References}

[1] Zhang Chuanneng. Research on isolation, purification and bioactivity of staphylococcus polysaccharides [D]. Fujian Normal University, 2015.

[2] Yi Sun Immunostimulatory and anti-tumor activities of a polysaccharide from Trametes robiniophila Murr. [J]. Carbohydrate Polymers, Volume 92, Issue 1, 30 January 2013, Pages 577-582.

[3] Yamamoto K, Kimura T, Sugitachi A, Matsuura N. Anti-angio-genic and anti-metastatic effects of beta- 1, 3- D- glucan purified from Hanabiratake, Sparassis crispa [J]. Biol Pharm Bull,2009, 32(2):259- 263.

[4] Wang Changsheng. Isolation, purification, structure identification and bioactivity test of the Achyranthes Bidentata Polysaccharides [D]. Guangdong Pharmaceutical University, 2016.

[5] Dong Wenxia, Liu Ping, Zhang Jingsheng, Wu Peipei, Yang Ge. The process optimization of the static separation and purification of inonotus obliquus polysaccharide by DEAE-52 cellulose [J]. Science and Technology of Food Industry, 2018, 39 (04): 154-158+165.

[6] NY/T 1676-2008, Measurement of crude polysaccharide content in edible fungus [S].

[7] Zheng Yushu, Zhao Pu, Zhao Hongkun. Improvement of SDS-PAGE staining - decolorization method [J]. Research and exploration in laboratory, 2007 (01): 41-42. 